\title{
BESO method for topology optimization of structures with high efficiency of heat dissipation
}

\author{
Dan $\mathrm{He}^{\mathrm{a}}$ and Shutian Liu \\ State Key Laboratory of Structutal Analysis for Industrial Equipment, Dept. of Engineering Mechanics, \\ Dalian Univerisity of Technology, 116023 Dalian, P.R. China
}

Received 22 August 2007, Accepted 11 February 2008

\begin{abstract}
The purpose of this paper is to present a Bi-Directional Evolutionary Structural Optimization (BESO) method for topology optimization of heat conduction structures. In BESO method the elements are allowed to be added as well as removed. Focused on the heat performance of structure, the additive criterion and rejection criterion were proposed respectively. With the limit volume of the high conductive material, the optimal layout of structure with high efficiency of heat dissipation and uniform temperature distribution can be obtained efficiently by BESO procedure.
\end{abstract}

\section{Introduction}

The design process is generally divided into two stages: conceptual design and detailed design. The structural layout can be obtained in the conceptual design stage. Then the shape and the size of the structure are acquired in the detailed design stage. It has been recognized that by using the topology optimization techniques in the conceptual design stage, we can yield substantial improvements in the performance of structures.

Relative to the shape and topology optimization of structures with mechanical properties (Bendsoe [1], Li et al. [2], Rozvany et al. [3]), the thermal conducting solid issue has received relatively less attention in spite of its significance. Cooling fins, thermal diffusers and moulding dies are examples of shape optimization in this category (Lee [4]). With the rapid development of microelectronic and micro processing technology, how to remove the heat as rapidly as possible from the package becomes further important. One solution is to insert a finite amount of high-conductivity material in the structure to increase the conductivity of the structure. Optimizing the topology (the distribution of the high conductivity material) can not only reduce the cost of high-conductivity material and manufacture, but also suit for further miniaturization (Shutian and Yongcun [5]). Bejan [6] puts forward the "tree-like network" construct method based on the construction theory which derived the optimal high effective conduction channel distribution with uniform heat resource and high conductivity ratio of high conductive material to conductivity of substrate material. Cheng et al. [7] constructed the high effective conduction channel in different conditions by bionic opti-

\footnotetext{
a Corresponding author:

stliu@dlut.edu.cn, hedan@student.dlut.edu.cn
}

mization based on the biological evolution principle. The bionic optimization has no limitations that the heat generation is uniform and the ratio of two kind's materials is high, however, the construct obtained by bionic optimization is similar to that obtained by Bejan's "tree-like network" construct method both in structure features and heat transfer performance. In $\mathrm{Wu}$ et al. [8], the "tree-like network" construct method was improved and the high effective conduction channel distribution was optimized again without the premise that the new order assembly construct must be assembled by the optimized last order construct. A better construct was obtained and the limit of the minimum heat resistance is derived when the thermal conductivity and the proportion of the two heat conduction materials are constant. Li et al. [9] developed an efficient finite element based computational procedure for the topology design of heat conducting fields. By removing or degenerating the conductive material of the elements with the most negative sensitivity the temperature objective at the control point can be most efficiently reduced.

The evolutionary structural optimization (ESO) method has been studied in recent years ( $\mathrm{Li}$ et al. [2]). Originally the method was conceived from the engineering perspective that the topology and shape of structures were naturally conservative for safety reasons and therefore contained an excess of material. To move from the conservative design to a more optimum design would therefore involve the removal of material. On the contrary, by adding material onto the most efficient regions begins from a minimum structure to obtain optimum is the additive ESO (AESO) method (Querin et al. [10]).

The validity of the ESO method has been examined critically and several arguments have been made against it (Zhou et al. [17], Rozvany et al. [3]), since it may lead to highly non-optimal solutions in same circumstances. 
However, this method could be justified if a user wishes to achieve rapid design improvement at a low computational cost. Recently, it has been stated that ESO has an actual theoretical basis (Tanskanen [18]).

A new development in ESO is the recent introduction of bidirectional evolutionary structural optimization (BESO), which is the combination of ESO and AESO. In BESO, elements are allowed to be added as well as removed (Querin et al. [11], Huang and Xie [12], Zhu et al. [13]). In recent years the ESO method has been developed into an effective engineering design tool and successfully extended allowing different structural constraints to be incorporated into the optimization process, such as stiffness (Chu et al. [14]), frequency (Li et al. [2]) or buckling (Manickarajah et al. [15]). Li et al. [16] extend the algorithm of Evolutionary Structural Optimization to shape and topology design problems subjected to steady heat conduction. Li et al. [9] developed an efficient finite element based computational procedure for the topology design of heat conducting fields. Based on the authors' knowledge, the Bi-Directional Evolutionary Structural Optimization (BESO) method has not been used in topology design for heat dissipation.

The purpose of this paper is to present a Bi-Directional Evolutionary Structural Optimization (BESO) method for topology optimization of structures with high efficiency of heat dissipation. The BESO method is the combination of ESO and AESO, which makes elements allowed to be added and rejected. So it has two evolution procedures: the AESO procedure and the ESO procedure. Considering the design object of high efficiency of heat dissipation and uniform distribution of temperature, sensitivity of heat resistance was taken as the element rejection criterion in the ESO procedure, which makes the efficiency of heat dissipation high; and the elemental temperature gradient level was taken as the element additive criterion which makes the temperature distribution of structure uniform.

The design domain of the structure is depicted by the relative density of high-conductivity material at any point and the relative densities assigned to the finite elements are chosen as design variables. Being similar with the compliance in structural problems we introduce a factor of heat resistance which is defined as the integration of the product of heat flux and temperature over the domain. Heat resistance generally described the efficiency of heat dissipation of structure. The element sensitivity of heat resistance was taken as element rejection criterion in the ESO procedure, which means the elements with lowly sensitivity of heat resistance are regarded as inefficient region and should be removed from structure. The elemental temperature gradient level was taken as the element additive criterion in the AESO procedure, which means elements with highly temperature gradient are considered as efficient region and should be added material on that. The checkerboard patterns (Bourdin [19], Zhou et al. [17]) for topology optimization of mechanical problems, can also be observed in heat conduction problems based on the ESO procedure. To avoid the checkerboard design, an intuitive smoothing technique is introduced. Numerical examples are also presented to demonstrate the capabilities of the method proposed in this paper.

\section{Heat resistance and sensitivity analysis}

The governing equation and boundary condition for general steady state heat conduction problems are well known as

$$
\begin{gathered}
\frac{\partial}{\partial \mathbf{x}_{i}}\left(\mathbf{k}_{i j} \frac{\partial \mathbf{T}}{\partial \mathbf{x}_{j}}\right)+\mathbf{Q}=0, \quad \text { on } \Omega \\
\left.\mathbf{T}\right|_{t=0}=\mathbf{T}_{\mathbf{0}}(x, y, z), \quad \text { on } \Gamma_{1} \\
\left(\alpha_{i} \frac{\partial \mathbf{T}}{\partial \mathbf{x}_{i}} n_{i}\right)_{s}=q_{s}, \text { on } \Gamma_{2} \\
\alpha_{i} \frac{\partial \mathbf{T}}{\partial \mathbf{x}_{i}} n_{i}=h\left(\mathbf{T}_{f}-\mathbf{T}\right), \text { on } \Gamma_{3} .
\end{gathered}
$$

Where $\mathbf{k}_{i j}$ denotes heat conductivities, $\mathbf{Q}$ the heat energy generated per unit volume, $\mathbf{T}$ the temperature field. Equation (2) described the temperature condition on boundary $\Gamma_{1}$, and $\mathbf{T}_{0}(x, y, z)$ denotes the given temperature. Equation (3) described the heat flux condition on boundary $\Gamma_{2}$, and $q_{s}$ the external heat flux. Equation (4) described the convectional condition on boundary $\Gamma_{3}$, and $h$ the convection heat transfer coefficient, and $T_{f}$ the temperature of coolant.

Equation (1) can be approximated by means of a finite element formulation as

$$
\mathbf{K T}-\mathbf{Q}=0
$$

where $\mathbf{K}$ represents the global conductivity matrix, $\mathbf{T}$ the global nodal temperature vector and $\mathbf{Q}$ the applied heat load vector.

To represent the efficiency of heat dissipation, a factor of heat resistance is introduced here, which is defined as the integration of the product of generating heat rate in unit volume $Q(x)$ and temperature $\bar{T}(x)$ over the domain, and it is chosen as the design objective function of the ESO procedure.

$$
D=\int_{\Omega} \overline{\mathbf{T}} \mathbf{Q} d \boldsymbol{\Omega}+\int_{\Gamma_{2}+\Gamma_{3}} q_{n} \overline{\mathbf{T}} d s
$$

When $Q$ is specific, reducing the temperature $T$ will lead to the reduction of the factor of heat resistance $D$. Generally, to minimize the temperature can be implemented by minimizing this factor $D$. In the other hand, the factor of heat resistance described the heat energy stored in the structures when the heat exchange process reaches the steady state. Thus, $D$ can be chosen as the represented index of the heat dissipation property of the structures. When the heat conduction problem is solved with finite 
element method, and only the temperature boundary condition is considered, the factor of heat resistance can be expressed in teams of the nodal temperature vector as

$$
D=\mathbf{T}^{\mathrm{T}} \mathbf{K} \mathbf{T} .
$$

Based on the basic idea of structural topology optimization, the topology of the structure is depicted by the relative density of materials at any point and the relative density assigned to each element is chosen as design variable (Bendsoe and Sigmund [20]).

$$
\rho_{i}=\left\{\begin{array}{ll}
1 & \text { if } \quad i \text { th element } \in \Omega_{s} \\
0 & \text { if } \quad i \text { th element } \in \Omega / \Omega_{s}
\end{array} \quad i=1,2, n_{e},\right.
$$

where $\rho_{i}$ denotes the density of the materials at $i$ th element, and the element which has $\rho_{i}=1$ means there high conductive material exists; the element which has $\rho_{i}=0$ means there a void exists. $\Omega$ denotes the design domain, $\Omega_{s}$ denotes the area occupied by solid material, and $n_{e}$ denotes the total number of elements in the design domain. The heat conductive matrix of each element $\mathbf{k}_{i}$ is linearly depended on the density of the $i$ th element, which is shown as:

$$
\mathbf{k}_{i}=\mathbf{k}_{i 0} \times \rho_{i}
$$

where $\mathbf{k}_{i 0}$ is the conductive matrix of the $i$ th element when it was an entity element.

The sensitivity of the factor of heat resistance of heat conduction problem can be obtained by differentiating equation (7).

$$
\frac{\partial D}{\partial \rho_{i}}=\frac{\partial \mathbf{T}^{T}}{\partial \rho_{i}} \mathbf{K} \mathbf{T}+\mathbf{T}^{T} \frac{\partial \mathbf{K}}{\partial \rho_{i}} \mathbf{T}+\mathbf{T}^{T} \mathbf{K} \frac{\partial \mathbf{T}}{\partial \rho_{i}} .
$$

Differentiating equation (5) yields

$$
\frac{\partial \mathbf{K}}{\partial \rho_{i}} \mathbf{T}+\mathbf{K} \frac{\partial \mathbf{T}}{\partial \rho_{i}}=0
$$

Introducing above equation into equation (9), the sensitivity of resistance factor can be expressed as

$$
\frac{\partial D}{\partial \rho_{i}}=-\mathbf{T}^{T} \frac{\partial \mathbf{K}}{\partial \rho_{i}} \mathbf{T}=-\mathbf{T}^{T} \mathbf{k}_{i 0} \mathbf{T}
$$

Thus, the sensitivity analysis of the objective function can be obtained mathematically and in an efficient manner in the iteration process.

\section{BESO method for topology optimization of heat conduction dissipation}

Here we present a BESO method for topology optimization of heat conduction dissipation. The BESO method is the combination of ESO and AESO, which makes elements allowable to be added and rejected. So it has two evolution procedures: the AESO procedure and the ESO procedure. Considering the design purpose being to find an appropriate distribution of high conductive material to improve the efficiency of heat dissipation and the fact that minimum difference of the maximum and minimum temperature in the domain is required, sensitivity of heat resistance was taken as the element rejection criterion in the ESO procedure, which makes the efficiency of heat dissipation high; and the elemental temperature gradient level was taken as the element additive criterion which makes the temperature distribution uniform.

\section{Element rejection criterion and the procedure of ESO}

It is clear that removing those conductive elements which have the lowest value of sensitivity of the factor of heat resistance will result in the least contribution to the addition of the energy of system. According the brief idea of ESO, by gradually rejecting the inefficient element, the optimal structure will be obtained. The evolutionary criterion for such a purpose is determined by comparing the heat resistance sensitivity of each element. So the element should be removed from domain field if its sensitivity of heat resistance is the lowest. The ESO procedure in each iteration was shown as follows:

(1) FEA analysis and sensitivity analysis;

(2) remove those entity elements which have the lowest value of sensitivity of heat resistance;

(3) calculate the numbers of element rejected in this iteration $\alpha$;

(4) if $\alpha<\alpha_{E S O}$, then repeat (1)-(4);

(5) if $\alpha \geqslant \alpha_{E S O}$, then finish this iteration of ESO.

Here the $\alpha_{E S O}$ was the element rejection ratio in each iteration, which is given to control the process of optimization. And by numerical experience, the ratio of element rejection was given as $\alpha_{E S O}=0.5 \%$, that's to say, there are $0.5 \%$ of all the elements in the domain field will be rejected during each iteration. Here the rejection of element doesn't means the density value was given as 0 , but the density of rejected element was given a very low value (as 1.0E-5) for the numerical continuity.

\section{Element additive criterion and the procedure of AESO}

Structure with best heat performance can be immediately described as: minimize the maximum temperature. Based on the intuitive thinking, the process of reducing the temperature as low as possible by adding material can be implemented by making the temperature distribution uniform. However, in the process of adding elements, it is best favoured for averaging the system temperature to add material onto the void element which has the largest temperature gradient to the next entity element.

The temperature of each element is described as the average value of the temperature of its nodes.

$$
T_{i}=\sum_{j=1}^{m} t_{j}
$$


where $T_{i}$ denotes the temperature of the $i$ th element, $t_{j}$ denotes the temperature of the $j$ th node of the element, and $m$ denotes the node number of the $i$ th element.

In the process of AESO, we compute the temperature gradient of every entity element and the next void element, choose the void element with largest temperature gradient to develop. In the optimize process, the temperature of entity element may be lower than the next void element. In that case, we compute the void element with highest temperature and make it as entity element, and then continue. The AESO procedure was shown as follows:

(1) FEA analysis;

(2) calculate the temperature of each element;

(3) calculate the temperature grad between entity element and its neighbour hole element;

(4) add material onto the hole which has the largest temperature gradient to the next entity element;

(5) calculate the number of element added in this iteration $\beta$;

(6) if $\beta<\beta_{A E S O}$, then repeat (1)-(6);

(7) if $\beta \geqslant \beta_{A E S O}$, then finish this iteration of AESO.

Here the $\beta_{A E S O}$ was the element added ratio in each iteration, which is given to control the process of optimization. By numerical experience, the ratio of element added was given as $\beta_{A E S O}=1.0 \%$, that's to say, there are $1.0 \%$ of all the elements in the domain field will be added during each iteration. Here the addition of element means the density of the hole element was changed to 1.0.

\section{Evolutionary procedure}

The BESO method is the combination of ESO and AESO, so in the BESO procedure, elements of the structure can either be added or removed. The evolutionary procedure was shown as follows:

(1) the maximum allowable domain the structure can occupy must be specified;

(2) the physical domain is subdivided with a densely populated regular finite element mesh, and all the elements are hole elements in initial;

(3) define all kinematic boundary constraints, loads, material properties and volume constraint;

(4) AESO procedure;

(5) ESO procedure;

(6) examine the volume constraint;

(7) repeat (4)-(6) until the volume constraint was reached.

What should be noticed is that, at the beginning of the optimization, all the elements are holes, we calculate the elements with highest temperature and develop them to entity elements in the first AESO process.

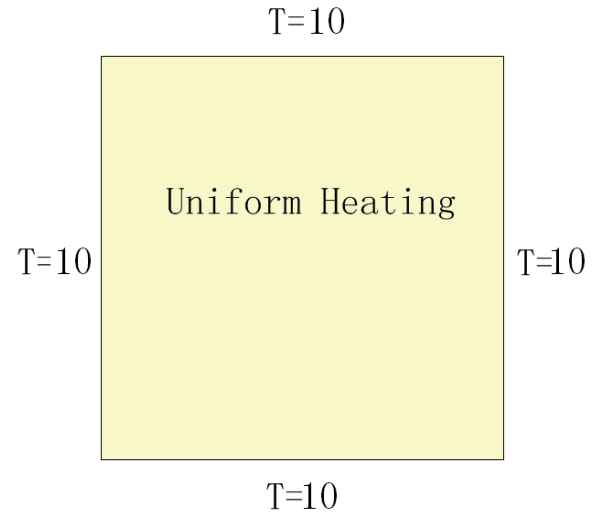

Fig. 1. A square plate with uniformly distributed heat resource and boundaries with prescribed temperatures.

\section{Smoothing technique}

The checkerboard patterns for topology optimization of mechanical problems, can also be observed in heat conduction problems based on the BESO procedure. To avoid the checkerboard design, an intuitive smoothing technique is introduced, in brief whose idea demonstrate as follow. The volume fraction of each element is affected by that of correlation elements, which reads as

$$
\begin{aligned}
& \overline{f_{s}^{m}}=\sum_{j=1}^{N} W_{m j} f_{s}^{m} \\
& \overline{f_{1}^{m}}=\sum_{j=1}^{N} W_{m j} f_{1}^{m} W_{m j}=\left(r_{0}-r_{m j}\right) / \sum_{k=1}^{N}\left(r_{0}-r_{m k}\right),
\end{aligned}
$$

where $N$ denotes total number of correlation elements to element $m$, which is determined by the factor $r_{0} . W_{m j}$ is the weight factor of element $j$ in element $m$, which is determined by the distance between centroids of two elements. The weight factor is shown as follows:

$$
W_{m j}=\left(r_{0}-r_{m j}\right) / \sum_{k=1}^{N}\left(r_{0}-r_{m k}\right),
$$

where $r_{m j}$ is the distance between the centroids of element $m$ and $j$.

\section{Illustrative examples}

Here we consider a square of plate (length of sides is $0.1 \mathrm{~m})$ with uniform heating resource $\left(3600 \mathrm{kw} / \mathrm{m}^{2}\right)$, and temperature of 4 sides were predicated $\left(10^{\circ} \mathrm{C}\right)$. The conductivity of base material is $k_{\text {low }}=2.1 \mathrm{w} /\left(\mathrm{m} .{ }^{\circ} \mathrm{C}\right)$, and the design target is to design the layout of high conductive material $\left(k_{h i g h}=210 \mathrm{w} /\left(\mathrm{m} .{ }^{\circ} \mathrm{C}\right)\right)$ on the plate which can reduce the temperature of structure and improve the efficiency of dissipation of structure.

The geometry model was shown in Figure 1, the volume constraint of the high conductivity material was $50 \%$. 


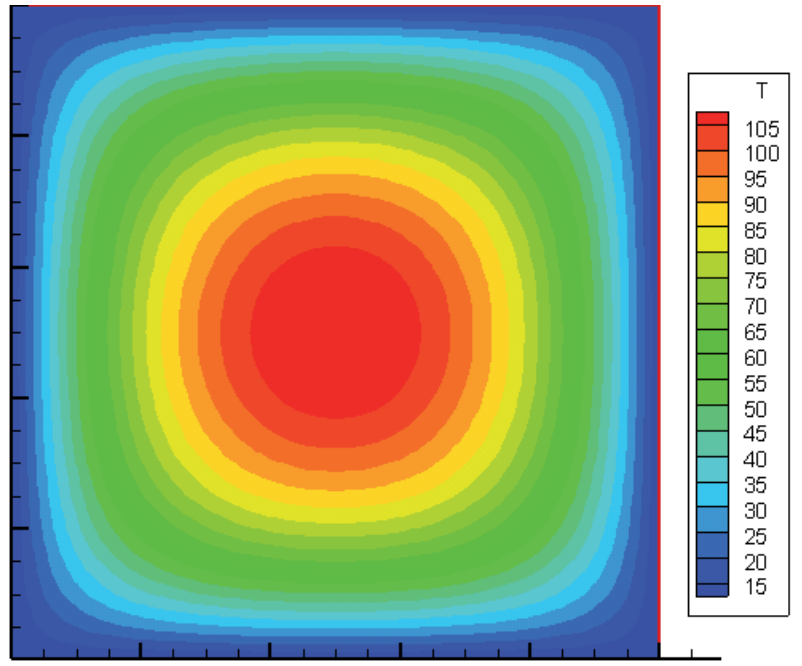

Fig. 2. Temperature distribution when high conductive material is uniformally distributed.

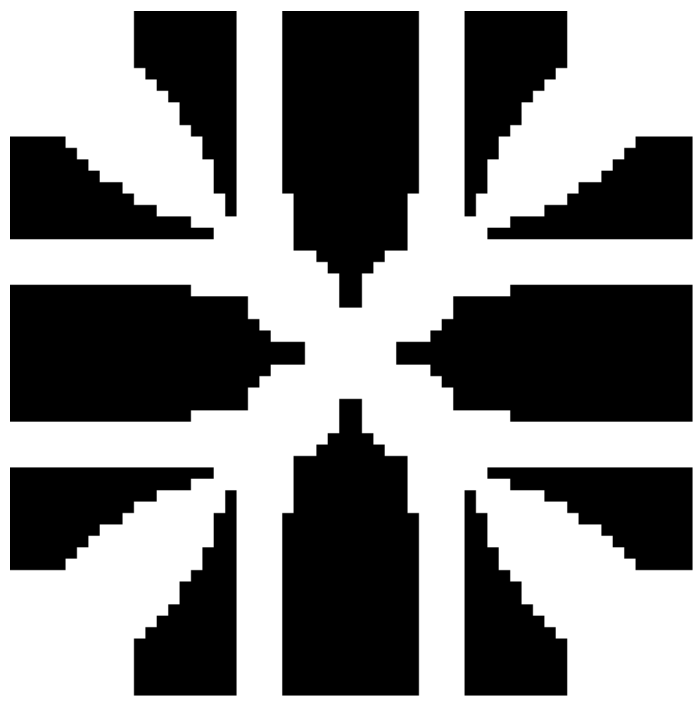

Fig. 3. Optimal distribution of high conductive material based on BESO method.

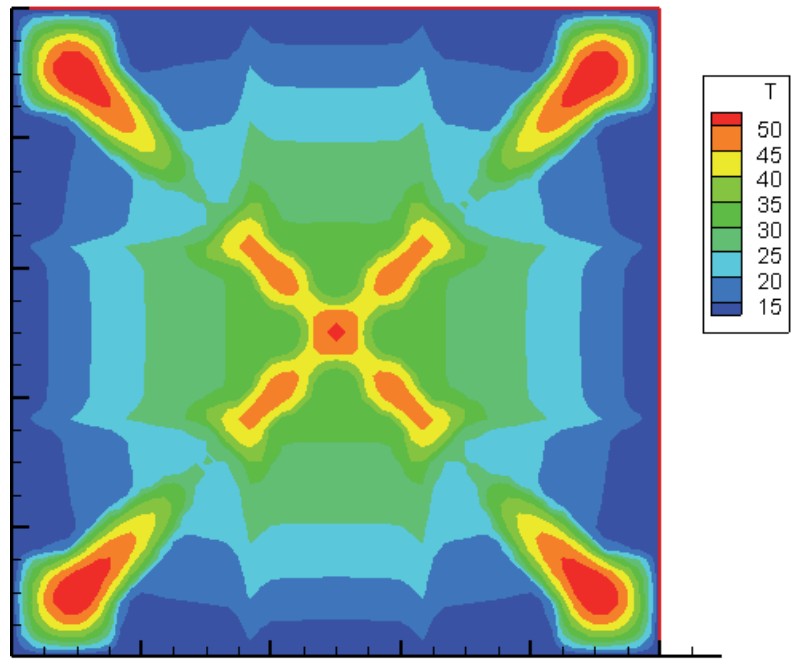

Fig. 4. Temperature distribution of optimal design based on BESO method.

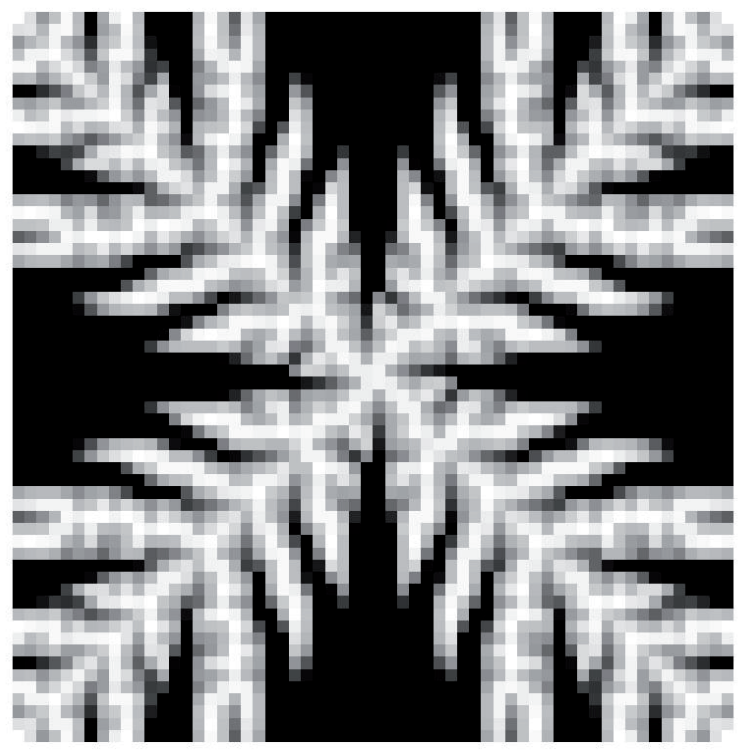

Fig. 5. Optimal distribution of high conductive material based on SIMP method.

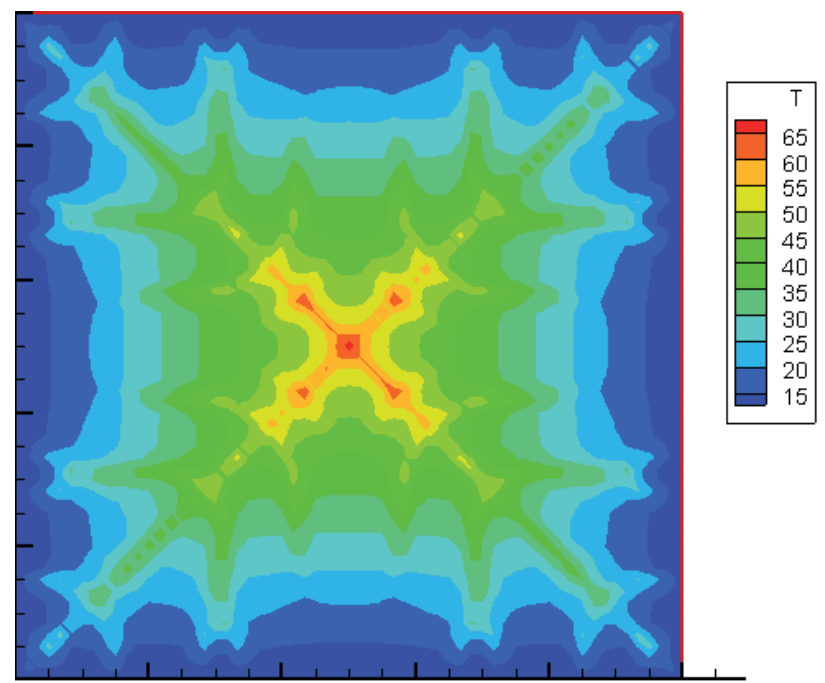

Fig. 6. Temperature distribution of optimal design based on SIMP method.

The design domain is divided into $60 \times 60=3600$ elements, and the layout of high conductive material was calculated by the proposed BESO method. The optimal result is shown in the Figure 3. The optimal results give out the path that collect and transfer the energy. From the temperature contour map shown in Figure 4, it can be seen that the temperature distribution is more uniformly distributed, and the maximum temperature is reduced about $50 \%$. And compare with the result based on SIMP method, the topology based on BESO method is similar with the optimal result based on SIMP method, which can demonstrate that the proposed method is valid. And the BESO result seemed more easily to manufacture. 


\section{Concluding remarks}

It can be concluded from this work that the topology optimization in the presence of heat conduction can be easily solved by the proposed BESO method. It can offer the designer with optimal solutions in the design stage which can make significant improvement in the thermal performance. The numerical experiments demonstrate that the proposed method is valid and effective. Compare with the result based on SIMP method, the topology based on BESO method is more easy to manufacture.

\section{References}

1. M.P. Bendsoe, Optimization of Structure Topology, Shape and Material, Springer-Verlag, Berlin (1995)

2. Q. Li, G.P. Steven, Y.M. Xie, A simple checkerboard suppression algorithm for evolutionary structural optimization. Structural and Multidisciplinary Optimization 22, 230-239 (2001)

3. G.I.N. Rozvany, M.P. Bendsoe, U. Kirsch, Layout optimization of structures. Applied Mechanics Review 48, 41118 (1995)

4. B.Y. Lee, Shape sensitivity formulation for an axisymmetric thermal conducting solids. J. Mech. Eng. Sci. 207, 209-216 (1993)

5. L. Shutian, Z. Yongcun, Design of high-conduction paths based on pology optimization, in Proc The Fourth China-Japan-Korea Joint Symposium on Optimization of Structural and Mechanics System Kunming, China (2006) pp. $449-454$

6. A. Bejan, Shape and Structure, from Engineering to Nature, Cambrige University Press (2000)

7. X.G. Cheng, Z.X. Li, Z.Y. Guo, Constructs of highly effective heat transport paths by bionic optimization. Science in China Series E-Technological Sciences 46, 296-302 (2003)

8. W.J. Wu, L.G. Chen, F.R. Sun, Improvement of tree-like network constructal method for heat conduction optimization. Science in China Series E-Technological Sciences 49, 332-341 (2006)
9. Q. Li, G.P. Steven, Y.M. Xie, O.M. Querin, Evolutionary topology optimization for temperature reduction of heat conducting fields. International Journal of Heat and Mass Transfer (2004) 47, 5071-5083

10. O.M. Querin, G.P. Steven, Y.M. Xie, Evolutionary structural optimisation using an additive algorithm. Finite Elements in Analysis and Design 34, 291-308 (2000)

11. O.M. Querin, V. Young, G.P. Steven, Y.M. Xie, Computational efficiency and validation of bi-directional evolutionary structural optimisation. Computer Methods in Applied Mechanics and Engineering 189, 559-573 (2000)

12. X. Huang, Y.M. Xie, Bidirectional evolutionary topology optimization for structures with geometrical and material nonlinearities. AIAA Journal 45, 308-313 (2007)

13. J.H. Zhu, W.H. Zhang, K.P. Qiu, Bi-directional evolutionary topology optimization using element replaceable method. Computational Mechanics 40, 97-109 (2007)

14. D.N. Chu, Y.M. Xie, A. Hira, G.P. Steven, Evolutionary structural optimization for problems with stiffness constraints. Finite Elements in Analysis and Design 21, 239251 (1996)

15. D. Manickarajah, Y.M. Xie, G.P. Steven, An evolutionary method for optimization of plate buckling resistance. Finite Elements in Analysis and Design 29, 205-230 (1998)

16. Q. Li, G.P. Steven, O.M. Querin, Y.M. Xie, Shape and topology design for heat conduction by evolutionary structural optimization. International Journal of Heat and Mass Transfer 42, 3361-3371 (1999)

17. M. Zhou, Y.K. Shyy, H.L. Thomas, Checkerboard and minimum member size control in topology optimization. Structural and Multidisciplinary Optimization 21 152$158(2001)$

18. P. Tanskanen, The evolutionary structural optimization method: theoretical aspects. Computer Methods in Applied Mechanics and Engineering 191, 5485-5498 (2002)

19. B. Bourdin, Filters in topology optimization. International Journal for Numerical Methods in Engineering 50, 2143-2158 (2001)

20. M.P. Bendsoe, O. Sigmund, Material interpolation schemes in topology optimization. Archive of Applied Mechanics 69, 635-654 (1999) 